Statement of Interest: None declared.

\section{REFERENCES}

1 Jaiis X, Olsson KM, Barbera JA, et al. Pregnancy outcomes in pulmonary arterial hypertension in the modern management era. Eur Respir J 2012; 40: 881-885.

2 Bendayan D, Hod M, Oron G, et al. Pregnancy outcome in patients with pulmonary arterial hypertension receiving prostacyclin therapy. Obstet Gynecol 2005; 106: 1206-1210.
3 Bonnin M, Mercier FJ, Sitbon O, et al. Severe pulmonary hypertension during pregnancy: mode of delivery and anesthetic management of 15 consecutive cases. Anesthesiology 2005; 102: 1133-1137.

4 Bédard E, Dimopoulos K, Gatzoulis MA. Has there been any progress made on pregnancy outcomes among women with pulmonary arterial hypertension? Eur Heart J 2009; 30: 256-265.

5 Kiely DG, Condliffe R, Webster V, et al. Improved survival in pregnancy and pulmonary hypertension using a multiprofessional approach. BJOG 2010; 117: 565-574.

DOI: $10.1183 / 09031936.00047512$

\title{
Chronic bronchitis: an objective diagnosis of exclusion
}

\section{To the Editors:}

In their recent, very interesting study, MONTES DE OCA et al. [1] suggested that chronic bronchitis is associated with increased morbidity and poorer general health status in both chronic obstructive pulmonary disease (COPD) and non-COPD subjects. Although the term "chronic bronchitis phenotype" is mentioned in the title, we feel that the differentiation between the phenotype and the actual diagnosis should be highlighted. Chronic bronchitis is a diagnosis of exclusion, while its phenotype could include patients with congestive heart failure (CHF), asthma, tuberculosis, lung cancer, bronchiectasis or other respiratory diseases. All these possible diagnoses were insufficiently investigated in this study. As a result, the overwhelming majority of non-COPD subjects with chronic bronchitis phenotype presented in this study are expected to match one of these diagnoses, with the well-established levels of morbidity and quality of life burden. We believe that the CHF diagnosis should have at least been inquired from all the subjects. In the COPD group, the same confounding factor should have been taken into account.

However, we believe that the prevalence of chronic bronchitis phenotype in COPD patients is underestimated in this study. First, we would like to note the fact that a forced expiratory volume in $1 \mathrm{~s} /$ forced vital capacity ratio of 0.7 is a cut point that lacks a physiological background. As a result, values near 0.7 can neither diagnose nor exclude COPD in the ignorance of the clinical context, especially in the elderly [2]. The significantly lower percentages of respiratory symptoms and of respiratory prescriptions in the nonbronchitic subgroup of COPD stage I patients also suggests a hyperdiagnosis of COPD in subjects without clinical disease. Moreover, the retrospective, subjective questionnaire in clinically stable patients could be misleading, since older people would more easily underestimate symptoms that are "expected for their age", whereas younger patients may overestimate symptoms that restrict their daily activities. This is compatible with the observation that COPD patients with chronic bronchitis show a general trend toward lower age and worse subjective symptoms, which cannot be explained otherwise. Age stratification could be enlightening.

\author{
Alexandros G. Mathioudakis*, Victoria Chatzimavridou- \\ Grigoriadou", Efstathia Evangelopoulou" and \\ Georgios A. Mathioudakis ${ }^{\#}$
}

*Medical Department, Macclesfield District General Hospital, National Health Service, Macclesfield, UK. "Respiratory Dept, General Hospital of Nikaia "St. Panteleimon", Piraeus, Greece.

Correspondence: A.G. Mathioudakis, Medical Dept, Macclesfield District General Hospital, National Health Service, Victoria Road, Macclesfield, SK10 3BL, UK. E-mail: a.mathioudakis@ nhs.net

Statement of Interest: None declared.

\section{REFERENCES}

1 Montes de Oca M, Halbert RJ, Lopez MV, et al. The chronic bronchitis phenotype in subjects with and without COPD: the PLATINO study. Eur Respir J 2012; 40: 28-36.

2 Vaz Fragoso CA, Concato J, McAvay G, et al. Chronic obstructive pulmonary disease in older persons: a comparison of two spirometric definitions. Respir Med 2010; 104: 1189-1196.

DOI: 10.1183/09031936.00107712

From the authors:

We would like to thank A.G. Mathioudakis and co-workers for their interest and comments regarding our recently published article in the July issue of the European Respiratory Journal [1].

A.G. Mathioudakis and co-workers raise a concern regarding the term of "chronic bronchitis phenotype" and the actual diagnosis. They also comment that the phenotype could include patients with tuberculosis, lung cancer, asthma, bronchiectasis and heart failure, and that these diagnoses were not sufficiently investigated in our study. We agree with the position that the phenotype includes patients with other conditions and, in this way, the term was addressed in our study [1]. The self-reported comorbidity (asthma, tuberculosis and lung cancer) of the subjects with 
spirometric diagnosis of chronic obstructive pulmonary disease (COPD) is clearly shown in table 3 of our article (description of subjects with COPD, by chronic bronchitis) [1]. However, we think that the problem is that A.G. Mathioudakis and co-workers did not understand the type of study PLATINO (Proyecto Latinoamericano de Investigación en Obstrucción Pulmonar) is. This study is a well-known population-based study on COPD and not a study of a selected COPD population, so it is not possible to conduct additional analyses to rule out, for example, congestive heart failure in this type of study. In addition, we think that it is important to highlight the existing proposal for defining COPD phenotypes as "a single or combination of disease attributes that describe differences between individuals with COPD as they relate to clinically meaningful outcomes (symptoms, exacerbations, response to therapy, rate of disease progression, or death)." This more focused definition allows classification of patients into distinct prognostic and therapeutic subgroups for both clinical and research purposes [2]. This definition is not restricted exclusively to selected COPD samples (there is no limit for its use in unselected populations), so it can also be used to study COPD phenotypes in a population-based sample and this is the case of our study. We presented the association of chronic bronchitis symptoms (a single attribute of the disease) in the PLATINO COPD population with the severity of airway obstruction, subjects' perceptions of their general health status, physical activity limitation and exacerbations (relation to clinically meaningful outcomes).

A.G. Mathioudakis and co-workers also made a comment regarding the low prevalence of the chronic bronchitis phenotype and suggest that this could be associated with the use of post-bronchodilator forced expiratory volume in $1 \mathrm{~s} /$ forced vital capacity ratio $<0.70$ to define COPD and the possible over-diagnosis of stage I COPD patients with low symptom prevalence. Unfortunately, it seems that A.G. Mathioudakis and co-workers have not reviewed the supplementary material of our study, which shows all analyses performed using the lower limit of normal (LLN) to define COPD [1]. Interestingly, supplemental tables 1 and 2 showed the parallel analysis using the LLN to define COPD, and the proportion of persons in both groups was quite similar to that found with the use of the fixed ratio [1]. Supplemental table 3 (description of subjects with COPD), and supplemental figures 1 and 2 (Global Initiative for Chronic Obstructive Lung Disease severity distribution of COPD subjects by chronic bronchitis and general health status assessed in COPD and non-COPD subjects, respectively) also showed the analyses using the LLN definition with similar findings [1].

Finally, we think that the data presented in our article helps to better understand the prevalence of the chronic bronchitis phenotype in an unselected COPD population using different criteria for defining COPD, and the association of this phenotype with some important outcomes (reduced pulmonary function, more respiratory symptoms and exacerbations, worse health status, and more physical activity limitation).

\section{María Montes de Oca* and Maria Victorina Lopez ${ }^{\#}$}

*Servicio de Neumonología, Hospital Universitario de Caracas, Facultad de Medicina, Universidad Central de Venezuela, Caracas, Venezuela. "Universidad de la República, Facultad de Medicina, Hospital Maciel, Montevideo, Uruguay.

Correspondence: M. Montes de Oca, Servicio de Neumonología, Piso 8, Hospital Universitario de Caracas, Universidad Central de Venezuela, Los Chaguaramos, 1030, Caracas, Venezuela. E-mail: mmdeoca@cantv.net.

Statement of Interest: None declared.

\section{REFERENCES}

1 Montes de Oca M, Halbert RJ, Lopez MV, et al. The chronic bronchitis phenotype in subjects with and without COPD: the PLATINO study. Eur Respir J 2012; 40: 28-36.

2 Han MK, Agusti A, Calverley PM, et al. Chronic obstructive pulmonary disease phenotypes: the future of COPD. Am J Respir Crit Care Med 2010; 182: 598-604.

DOI: $10.1183 / 09031936.00129812$ 